\title{
Dworkin's Paradox
}

Seung Ki Baek ${ }^{1}$, Jung-Kyoo Choi², Beom Jun Kim,*

1 Integrated Science Laboratory, Umeå University, S-901 87 Umeå, Sweden (Present address: School of Physics, Korea Institute for Advanced Study, Seoul 130-722, Korea) 2 School of Economics and Trade, Kyungpook National University, Daegu 702-701, Korea 3 BK21 Physics Research Division and Department of Physics, Sungkyunkwan University, Suwon 440-746, Korea

* E-mail: beomjun@skku.edu

\section{Derivation of the affinity function}

Let us consider two arbitrary distributions, $\boldsymbol{p}=\left(p_{1}, \ldots, p_{N}\right)$ and $\boldsymbol{q}=\left(q_{1}, \ldots, q_{N}\right)$, with $p_{i} \geq 0, q_{i} \geq 0$, and $\sum_{i}^{N} p_{i}=\sum_{i}^{N} q_{i}=1$. We will define an affinity function $\rho_{N}(\boldsymbol{p}, \boldsymbol{q})$ between them, whose specific functional form will be characterized by requiring the following postulates.

- P1: Separability

$$
\begin{aligned}
& \rho_{N}\left(\begin{array}{c}
p_{1}, \ldots, p_{N} \\
q_{1}, \ldots, q_{N}
\end{array}\right)=\rho_{N-k+1}\left(\begin{array}{l}
P_{k}, p_{k+1}, \ldots, p_{N} \\
Q_{k}, q_{k+1}, \ldots, q_{N}
\end{array}\right) \\
& +\Delta\left(P_{k}, Q_{k}\right)\left[\rho_{k}\left(\begin{array}{c}
\frac{p_{1}}{P_{k}}, \ldots, \frac{p_{k}}{P_{k}} \\
\frac{q_{k}}{Q_{k}}, \ldots, \frac{q_{k}}{Q_{k}}
\end{array}\right)-1\right],
\end{aligned}
$$

where $P_{k} \equiv p_{1}+p_{2}+\cdots+p_{k}$ and $Q_{k} \equiv q_{1}+q_{2}+\cdots+q_{k}$ are positive with $1<k<N$. In addition, $\Delta\left(P_{k}, Q_{k}\right)$ is a non-negative differentiable function and converges to zero as $P_{k} \rightarrow 0$ or $Q_{k} \rightarrow 0$. The situation can be described as follows: we first observe $\mathbf{p}^{\prime}=\left(P_{k}, p_{k+1}, \ldots, p_{N}\right)$ and $\mathbf{q}^{\prime}=\left(Q_{k}, q_{k+1}, \ldots, q_{N}\right)$, instead of the real $\mathbf{p}$ and $\mathbf{q}$. We can calculate affinity $\rho_{N-k+1}$ in this resolution. The question is how affinity will change when we come to know the real $\mathbf{p}$ and $\mathbf{q}$. If the substructures inside $P_{k}$ and $Q_{k}$ have an exact affinity by $\rho_{k}=1$, nothing will change from the previous calculation, i.e., $\rho_{N}=\rho_{N-k+1}$. If they actually had no affinity by $\rho_{k}=0$ in this better resolution, on the other hand, the overall affinity should decrease by a certain amount $\Delta$, which will be a function of $P_{k}$ and $Q_{k}$. For example, for $P_{k} \ll 1$ or $Q_{k} \ll 1$, the decrement in $\rho_{N}$ will be also vanishingly small even if the subpopulations inside them look completely different.

- P2: Invariance under permutation

$$
\rho_{3}\left(\begin{array}{c}
p_{1}, p_{2}, p_{3} \\
q_{1}, q_{2}, q_{3}
\end{array}\right)=\rho_{3}\left(\begin{array}{c}
p_{2}, p_{1}, p_{3} \\
q_{2}, q_{1}, q_{3}
\end{array}\right)=\ldots=\rho_{3}\left(\begin{array}{c}
p_{3}, p_{2}, p_{1} \\
q_{3}, q_{2}, q_{1}
\end{array}\right) .
$$

Note that it is for $N=3$, which is enough to prove the same property for general $N$ in combination with the other postulates.

- P3: Non-negativity

$$
\rho_{N}(\boldsymbol{p}, \boldsymbol{q}) \geq 0,
$$

where $\rho_{N}(\boldsymbol{p}, \boldsymbol{q})=0$ if and only if $\boldsymbol{p}$ is orthogonal to $\boldsymbol{q}$, whereas a maximum value is obtained if and only if $\boldsymbol{p}=\boldsymbol{q}$.

- P4: Symmetry

$$
\rho_{N}(\boldsymbol{p}, \boldsymbol{q})=\rho_{N}(\boldsymbol{q}, \boldsymbol{p})
$$


Let us explain some direct consequences of P1. For $N=2$, it yields a trivial equality. For $N=3$, we have

$$
\rho_{3}\left(\begin{array}{c}
p_{1}, p_{2}, p_{3} \\
q_{1}, q_{2}, q_{3}
\end{array}\right)=\rho_{2}\left(\begin{array}{c}
P_{2}, p_{3} \\
Q_{2}, q_{3}
\end{array}\right)+\Delta\left(P_{2}, Q_{2}\right)\left[\rho_{2}\left(\begin{array}{c}
\frac{p_{1}}{P_{2}}, \frac{p_{2}}{P_{2}} \\
\frac{q_{1}}{Q_{2}}, \frac{q_{2}}{Q_{2}}
\end{array}\right)-1\right] .
$$

For $N=4$, we will see two different ways to calculate affinity between $\left(P_{3}, p_{4}\right)$ and $\left(Q_{3}, q_{4}\right)$. The first way is the following:

$$
\begin{aligned}
& \rho_{4}\left(\begin{array}{c}
p_{1}, \ldots, p_{4} \\
q_{1}, \ldots, q_{4}
\end{array}\right) \\
= & \rho_{2}\left(\begin{array}{c}
P_{3}, p_{4} \\
Q_{3}, q_{4}
\end{array}\right)+\Delta\left(P_{3}, Q_{3}\right)\left[\rho_{3}\left(\begin{array}{c}
\frac{p_{1}}{P_{3}}, \frac{p_{2}}{P_{3}}, \frac{p_{3}}{P_{3}} \\
\frac{q_{3}}{Q_{3}}, \frac{q_{2}}{Q_{3}}, \frac{q_{3}}{Q_{3}}
\end{array}\right)-1\right] \\
= & \rho_{2}\left(\begin{array}{c}
P_{3}, p_{4} \\
Q_{3}, q_{4}
\end{array}\right)+\Delta\left(P_{3}, Q_{3}\right)\left\{\rho_{2}\left(\begin{array}{c}
\frac{P_{2}}{P_{3}}, \frac{p_{3}}{P_{3}} \\
\frac{Q_{2}}{Q_{3}}, \frac{q_{3}}{Q_{3}}
\end{array}\right)\right. \\
& \left.+\Delta\left(\frac{P_{2}}{P_{3}}, \frac{Q_{2}}{Q_{3}}\right)\left[\rho_{2}\left(\begin{array}{c}
\frac{p_{1}}{P_{2}}, \frac{p_{2}}{P_{2}} \\
\frac{q_{1}}{Q_{2}}, \frac{q_{2}}{Q_{2}}
\end{array}\right)-1\right]-1\right\} .
\end{aligned}
$$

We then consider another way to calculate the same quantity as follows:

$$
\begin{aligned}
& \rho_{4}\left(\begin{array}{c}
p_{1}, \ldots, p_{4} \\
q_{1}, \ldots, q_{4}
\end{array}\right) \\
= & \rho_{3}\left(\begin{array}{c}
P_{2}, p_{3}, p_{4} \\
Q_{2}, q_{3}, q_{4}
\end{array}\right)+\Delta\left(P_{2}, Q_{2}\right)\left[\rho_{2}\left(\begin{array}{c}
\frac{p_{1}}{P_{2}}, \frac{p_{2}}{P_{2}} \\
\frac{q_{1}}{Q_{2}}, \frac{q_{2}}{Q_{2}}
\end{array}\right)-1\right] \\
= & \rho_{2}\left(\begin{array}{l}
P_{3}, p_{4} \\
Q_{3}, q_{4}
\end{array}\right)+\Delta\left(P_{3}, Q_{3}\right)\left[\rho_{2}\left(\begin{array}{c}
\frac{P_{2}}{P_{3}}, \frac{p_{3}}{P_{3}} \\
\frac{Q_{2}}{Q_{3}}, \frac{q_{3}}{Q_{3}}
\end{array}\right)-1\right] \\
& +\Delta\left(P_{2}, Q_{2}\right)\left[\rho_{2}\left(\begin{array}{c}
\frac{p_{1}}{P_{2}}, \frac{p_{2}}{P_{2}} \\
\frac{q_{1}}{Q_{2}}, \frac{q_{2}}{Q_{2}}
\end{array}\right)-1\right] .
\end{aligned}
$$

Comparing Eq. (1) with Eq. (2), we see that

$$
\Delta\left(P_{3}, Q_{3}\right) \Delta\left(\frac{P_{2}}{P_{3}}, \frac{Q_{2}}{Q_{3}}\right)=\Delta\left(P_{2}, Q_{2}\right) .
$$

Note that $P_{2} / P_{3}$ can take any value between zero and one, regardless of $P_{3}$, and that $Q_{2} / Q_{3}$ is also independent of $Q_{3}$ in the same way, as long as $P_{3}$ and $Q_{3}$ are nonzero.

Now we define

$$
g(x, y) \equiv \rho_{2}\left(\begin{array}{l}
x, 1-x \\
y, 1-y
\end{array}\right)-1
$$

for $x, y \in[0,1]$. From $\mathrm{P} 1$ and $\mathrm{P} 2$, it is straightforward to verify

$$
g(x, y)=g(1-x, 1-y)
$$

since

$$
\begin{aligned}
& \rho_{3}\left(\begin{array}{c}
p_{1}, p_{2}, p_{3} \\
q_{1}, q_{2}, q_{3}
\end{array}\right)=\rho_{3}\left(\begin{array}{c}
p_{2}, p_{1}, p_{3} \\
q_{2}, q_{1}, q_{3}
\end{array}\right) \\
= & \rho_{2}\left(\begin{array}{c}
P_{2}, p_{3} \\
Q_{2}, q_{3}
\end{array}\right)+\Delta\left(P_{2}, Q_{2}\right)\left[\rho_{2}\left(\begin{array}{c}
\frac{p_{1}}{P_{2}}, \frac{p_{2}}{P_{2}} \\
\frac{q_{1}}{Q_{2}}, \frac{q_{2}}{Q_{2}}
\end{array}\right)-1\right] \\
= & \rho_{2}\left(\begin{array}{c}
P_{2}, p_{3} \\
Q_{2}, q_{3}
\end{array}\right)+\Delta\left(P_{2}, Q_{2}\right)\left[\rho_{2}\left(\begin{array}{c}
\frac{p_{2}}{P_{2}}, \frac{p_{1}}{P_{2}} \\
\frac{q_{2}}{Q_{2}}, \frac{q_{1}}{Q_{2}}
\end{array}\right)-1\right] .
\end{aligned}
$$


Applying P1 to another permutation

$$
\rho_{3}\left(\begin{array}{c}
p_{1}, p_{2}, p_{3} \\
q_{1}, q_{2}, q_{3}
\end{array}\right)=\rho_{3}\left(\begin{array}{c}
p_{3}, p_{2}, p_{1} \\
q_{3}, q_{2}, q_{1}
\end{array}\right),
$$

we are directly led to

$$
\begin{aligned}
& g\left(p_{3}, q_{3}\right)+\Delta\left(1-p_{3}, 1-q_{3}\right) g\left(\frac{p_{1}}{1-p_{3}}, \frac{q_{1}}{1-q_{3}}\right) \\
= & g\left(p_{1}, q_{1}\right)+\Delta\left(1-p_{1}, 1-q_{1}\right) g\left(\frac{p_{3}}{1-p_{1}}, \frac{q_{3}}{1-q_{1}}\right),
\end{aligned}
$$

where $p_{1}, p_{3}, q_{1}, q_{3} \in[0,1)$. Let us redefine the variables as $\frac{p_{1}}{1-p_{3}} \equiv p, \frac{q_{1}}{1-q_{3}} \equiv q, 1-p_{3} \equiv r$, and $1-q_{3} \equiv s$. Each of these new variables $p, q, r$, and $s$ can take an arbitrary value independently of one another, as long as $p, q \in[0,1]$ and $r, s \in(0,1)$. The above equality can be then rewritten as

$$
\begin{gathered}
g(r, s)+\Delta(r, s) g(p, q) \\
=g(p r, q s)+\Delta(1-p r, 1-q s) g\left(\frac{1-r}{1-p r}, \frac{1-s}{1-q s}\right) .
\end{gathered}
$$

Defining a function

$$
f(p, q, r, s) \equiv g(r, s)+[\Delta(r, s)+\Delta(1-r, 1-s)] g(p, q)
$$

for $p, q, r, s \in(0,1)$, we apply Eq. (6) to $f(p, q, r, s)$ to obtain

$$
\begin{aligned}
f(p, q, r, s)= & g(p r, q s) \\
& +\Delta(1-p r, 1-q s) g\left(\frac{1-r}{1-p r}, \frac{1-s}{1-q s}\right) \\
& +\Delta(1-r, 1-s) g(p, q) .
\end{aligned}
$$

Note from Eq. (3) that

$$
\begin{aligned}
& \Delta\left(p_{2}+p_{3}, q_{2}+q_{3}\right) \Delta\left(\frac{p_{3}}{p_{2}+p_{3}}, \frac{q_{3}}{q_{2}+q_{3}}\right) \\
= & \Delta(1-p r, 1-q s) \Delta\left(\frac{1-r}{1-p r}, \frac{1-s}{1-q s}\right) \\
= & \Delta(1-r, 1-s) .
\end{aligned}
$$

Hence, we see that

$$
\begin{aligned}
& f(p, q, r, s) \\
= & g(p r, q s)+\Delta(1-p r, 1-q s) g\left(\frac{1-r}{1-p r}, \frac{1-s}{1-q s}\right) \\
& +\Delta(1-p r, 1-q s) \Delta\left(\frac{1-r}{1-p r}, \frac{1-s}{1-q s}\right) g(p, q) \\
= & g(p r, q s)+\Delta(1-p r, 1-q s)\left[g\left(\frac{1-r}{1-p r}, \frac{1-s}{1-q s}\right)\right. \\
& \left.+\Delta\left(\frac{1-r}{1-p r}, \frac{1-s}{1-q s}\right) g(p, q)\right] \\
= & g(p r, q s)+\Delta(1-p r, 1-q s)\left\{g\left[\frac{p(1-r)}{1-p r}, \frac{q(1-s)}{1-q s}\right]\right. \\
& \left.+\Delta\left(\frac{1-p}{1-q r}, \frac{1-q}{1-q s}\right) g(r, s)\right\},
\end{aligned}
$$


where the last equality comes from Eq. (6). Equation (5) then gives us

$$
g\left[\frac{p(1-r)}{1-p r}, \frac{q(1-s)}{1-q s}\right]=g\left(\frac{1-p}{1-p r}, \frac{1-q}{1-q s}\right),
$$

relating Eqs. (8) and (9) as follows:

$$
\begin{aligned}
& f(p, q, r, s) \\
= & g(p r, q s)+\Delta(1-p r, 1-q s)\left[g\left(\frac{1-r}{1-p r}, \frac{1-s}{1-q s}\right)\right. \\
& \left.+\Delta\left(\frac{1-r}{1-p r}, \frac{1-s}{1-q s}\right) g(p, q)\right] \\
= & g(p r, q s)+\Delta(1-p r, 1-q s)\left[g\left(\frac{1-p}{1-p r}, \frac{1-q}{1-q s}\right)\right. \\
& \left.+\Delta\left(\frac{1-p}{1-p r}, \frac{1-q}{1-q s}\right) g(r, s)\right] .
\end{aligned}
$$

In short, we have just confirmed that

$$
f(p, q, r, s)=f(r, s, p, q) .
$$

From the definition of $f(p, q, r, s)$ in Eq. (7), we see that

$$
\frac{g(r, s)}{g(p, q)}=\frac{\Delta(r, s)+\Delta(1-r, 1-s)-1}{\Delta(p, q)+\Delta(1-p, 1-q)-1}
$$

For this to be true for every independent set of $(p, q, r, s)$, it must be that

$$
g(r, s)=C[\Delta(r, s)+\Delta(1-r, 1-s)-1]
$$

with a certain constant $C$. Then P1 says that

$$
\lim _{\substack{r \rightarrow 0 \\ s \rightarrow 1}} g(r, s)=-C,
$$

which is the lowest possible value of $g(r, s)$. However, according to the definition of $g(r, s)$ in Eq. (4) and P3, this lower bound should be -1 . In other words, $C=1$ and

$$
\rho_{2}\left(\begin{array}{l}
r, 1-r \\
s, 1-s
\end{array}\right)=\Delta(r, s)+\Delta(1-r, 1-s)
$$

We have restricted our variables as $r \in(0,1)$ and $s \in(0,1)$, but it is not difficult to check that the above expression can be readily used in $r \in[0,1]$ and $s \in[0,1]$ when $\Delta$ is characterized as will be discussed below. Then $\mathrm{P} 1$ works as a recursive relation, yielding an expression for general $N$ :

$$
\rho_{N}=\sum_{i=1}^{N} \Delta\left(p_{i}, q_{i}\right) .
$$

Now we ask ourselves how $\Delta$ should look. P1 and P4 imply that $\Delta$ can be expanded as $\Delta\left(p_{i}, q_{i}\right)=$ $c_{1}\left(p_{i} q_{i}\right)^{\alpha_{1}}+c_{2}\left(p_{i} q_{i}\right)^{\alpha_{2}}+\cdots$ with coefficients $c_{n}$ and exponents $\alpha_{n}>0$. So let us write

$$
\rho_{N}=\sum_{i=1}^{N} \sum_{n=1}^{\infty} c_{n}\left(p_{i} q_{i}\right)^{\alpha_{n}} .
$$


If we define $l_{\alpha}(\boldsymbol{p}, \boldsymbol{q}) \equiv \sum_{i=1}^{N}\left(p_{i} q_{i}\right)^{\alpha}$, it means that $\rho_{N}$ should be of the following form,

$$
\rho_{N}(\boldsymbol{p}, \boldsymbol{q})=\sum_{n=1}^{\infty} c_{n} l_{\alpha_{n}}(\boldsymbol{p}, \boldsymbol{q})
$$

Among every possible $l_{\alpha}$, it is only $l_{1 / 2}$ that satisfies the maximization condition in P3. This can be shown by variational calculus using a Lagrange multiplier $\mu$,

$$
\frac{\partial}{\partial q_{i}}\left[l_{\alpha}(\boldsymbol{p}, \boldsymbol{q})-\mu \sum_{j=1}^{N} q_{j}\right]_{q_{i}=p_{i}}=\alpha p_{i}^{2 \alpha-1}-\mu=0,
$$

which is satisfied for any $p_{i}$ when $\alpha=1 / 2$ and $\mu=\alpha$. Hence we separate this term from the others in Eq. (11) as

$$
\rho_{N}(\boldsymbol{p}, \boldsymbol{q})=c l_{1 / 2}(\boldsymbol{p}, \boldsymbol{q})+\sum_{n}^{\prime} c_{n} l_{\alpha_{n}}(\boldsymbol{p}, \boldsymbol{q}),
$$

where $\sum^{\prime}$ means that $\alpha_{n}=1 / 2$ is excluded from the summation. There exists a maximum value in $\rho_{N}$, which is obtained by

$$
\begin{aligned}
\rho_{N}(\boldsymbol{p}, \boldsymbol{p}) & =c l_{1 / 2}(\boldsymbol{p}, \boldsymbol{p})+\sum_{n}^{\prime} c_{n} l_{\alpha_{n}}(\boldsymbol{p}, \boldsymbol{p}) \\
& =c+\sum_{n}^{\prime} c_{n} \sum_{i} p_{i}^{2 \alpha_{n}},
\end{aligned}
$$

noting that $l_{1 / 2}(\boldsymbol{p}, \boldsymbol{p})=\sum_{i} p_{i}=1$. Since this value is the same for every $\boldsymbol{p}$, the last term should be kept constant for any $p_{i}$, and we have to choose $c_{n}=0$ for every $n$ in the second term. To sum up, our affinity function is characterized as

$$
\rho_{N}(\boldsymbol{p}, \boldsymbol{q})=c \sum_{i=1}^{N}\left(p_{i} q_{i}\right)^{1 / 2}
$$

with a positive constant $c$, the maximum value of $\rho_{N}$. 